\title{
Outreach and educational activities within the EEE cosmic ray network
}

\section{Chiara Pinto ${ }^{a, b, *}$ on behalf of the EEE Collaboration}

(a complete list of authors can be found at the end of the proceedings)

${ }^{a}$ Dipartimento di Fisica e Astronomia, Università degli Studi di Catania,

Via S. Sofia 64, Catania, Italy

${ }^{b}$ INFN Sezione di Catania,

Via S. Sofia 64, Catania, Italy

E-mail: chiara.pinto@ct.infn.it

The Extreme Energy Events (EEE) network consists in a sparse array of telescopes based on Multigap Resistive Plate Chambers, installed in high school buildings all over the Italian territory and at CERN. Besides the many research activities concerned with extensive air shower detection, long distance correlation studies and additional physics results obtained during the last decade, the EEE project is extensively employed for educational and outreach activities, constituting a unique opportunity to promote a fruitful and close collaboration between students, high-school teachers and researchers. The involvement is at all levels, from the construction of the chambers during short stages at CERN over the past 15 years, with the participation of several hundred high-school students and teachers, to the installation, monitoring and data taking with the telescopes by highschool teams, to masterclasses, physics lectures, data analysis sessions and joint discussions on the results and their interpretation. Recent developments of the EEE network led to the installation and use of additional detectors in the Arctic region and on board of sailing ships, to measure the cosmic ray flux over large latitude intervals. Periodical remote and in presence (pre-Covid era) meetings allowed in these years a large participation (several thousand people) from the high-school community to the EEE activities. National and local outreach initiatives in cosmic ray physics are also carried out around Italy by the EEE network, as a contribution to the dissemination of science among young people.

$37^{\text {th }}$ International Cosmic Ray Conference (ICRC 2021)

July 12 th - 23rd, 2021

Online - Berlin, Germany

\footnotetext{
*Presenter
} 


\section{Introduction}

The Extreme Energy Events (EEE) project [1] is a large array of particle detectors installed in Italian high-school buildings and physics laboratories spanning an area of more than $10^{5} \mathrm{~km}^{2}$, from CERN to Sicily. The experiment aims to study cosmic radiation by detecting and tracking the muon component of the Extensive Air Showers (EASs) produced by the interaction of high energy primary cosmic rays in the Earth atmosphere. The project has a large educational impact, with the involvement of thousands of students and high-school teachers every year. The detectors of the network are made of three Multigap Resistive Plate Chambers (MRPCs), that have been built at CERN by high-school teams under the supervision of EEE researchers.

The very good performance of the EEE detectors allows a large physics program, with the aim to investigate several aspects of the secondary muon flux, including the search for anisotropies of the secondary component at the sub-TeV scale [2], the extensive air showers detection [3, 4], the measurement of upward-going particles [5], the long distance correlation studies among detectors located hundred kilometres apart [6,7], several performance and simulation tools developments [8-11], the study of solar phenomena (such as the Forbush descreases [12, 13]), and also several applications of cosmic ray physics [14]. However, the milestone of the EEE activity is its educational mission. Indeed, the EEE project aims to provide a full research experience to high-school students, involving them from the construction of MRPCs to the data analysis, monitoring and maintenance of the detectors. Analyzing data, comparing and discussing results with other high-school fellows allow the students a glimpse of how professional scientific research works.

\section{Overview of the EEE activities with high-schools}

The EEE project started in 2004, funded and operated by the Museo Storico della Fisica e Centro Studi e Ricerche Enrico Fermi in Rome, in collaboration with CERN, INFN and MIUR. The initiative involves more than 100 educational institutes. About 60 high-schools distributed accross Italy host a detector, while others are involved in the project participating in data taking and analysis. The MRPCs are built by high-school teams, which are supervised by EEE researchers through the construction of sophisticated high precision detectors (see Fig. 1). This task is accomplished at CERN, which is made open to students specifically for this purpose. Afterwards, the telescopes are sent and installed in Italian high-schools, where students and teachers take care of the commissioning of the station and of the start of data taking operations. Students have also the fundamental task to monitor the status of the telescope installed in their school. Further details concerning the detector features are given in Section 3.

All the students of the project are involved in several meetings and conferences. On a monthly basis, students attend a Run Coordination Meeting, in which they can present updates on the ongoing analyses and discuss tests and detector updates. Every six months, students and teachers involved in the project are invited to conferences usually held at Ettore Majorana Foundation And Centre For Scientific Culture in Erice (Sicily), where masterclasses, measurement campaigns and general lectures on cosmic ray physics are organized.

Further information about the outreach activities carried out within the EEE project are given in Section 4. 

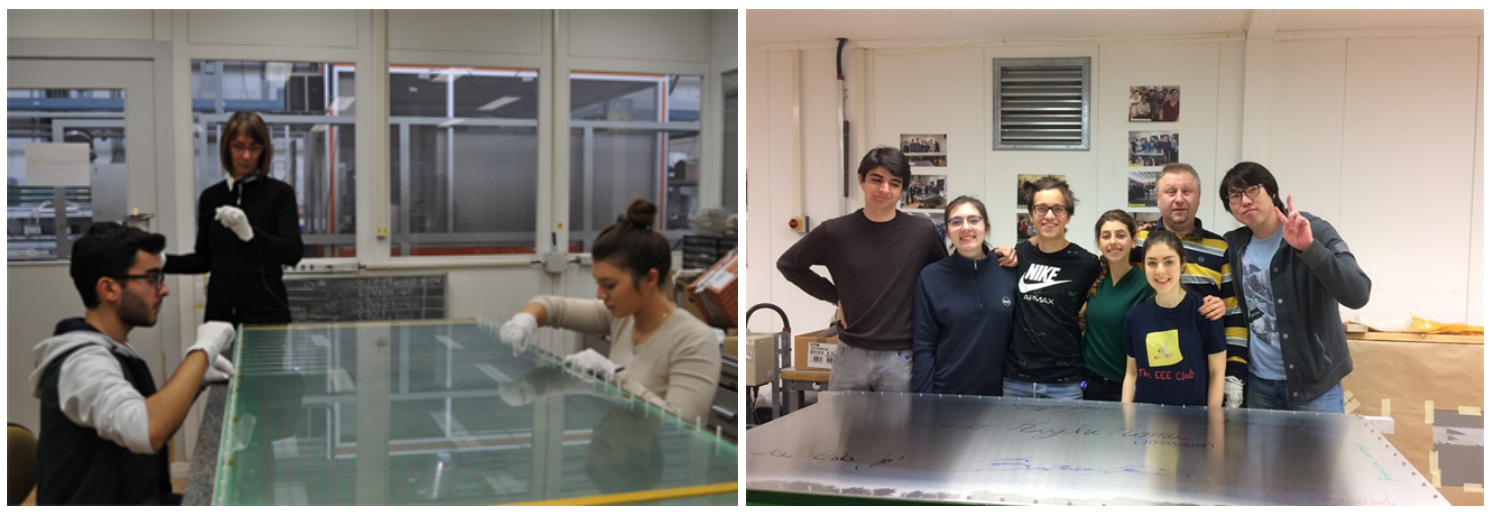

Figure 1: Pictures of high-school students of the project during the construction of the MRPCs of EEE telescopes, at CERN.

\section{Detector construction and operation}

The basic structure of each EEE telescope includes three MRPCs, each with a sensitive area of about $80 \times 160 \mathrm{~cm}^{2}$ and with six gas gaps, obtained by a stack of glass sheets, separated by narrow $(300 \mu \mathrm{m})$ gaps, assembled by high-school students at CERN. A typical EEE telescope and a sketch of the chamber's inner structure are shown in Fig. 2. The chambers are operated with high voltage (around $20 \mathrm{kV}$ ), applied only to the external glasses, coated with resistive paint. Chambers are usually operated with a gas mixture of $98 \% / 2 \%$ of $\mathrm{C}_{2} \mathrm{H}_{2} \mathrm{~F}_{4}$ and $\mathrm{SF}_{6}$, with a continuous flow and at the atmospheric pressure. The readout panel is split in 24 copper strips. The anode and cathode strips collect the signals induced by the particles, providing position information along one direction. The information along the other coordinate is obtained by measuring the time delay between the signals arriving at the two ends of a strip. The EEE telescopes have very good performance [15] both in terms of spatial resolution ( $\sigma_{\text {longitudinal }} \sim 1.5 \mathrm{~cm}, \sigma_{\text {transverse }} \sim 1.0 \mathrm{~cm}$ ) and of time resolution, $\sigma_{\text {time }} \sim 240$ ps. For most of the chambers, the detection efficiency is better than $90 \%$.

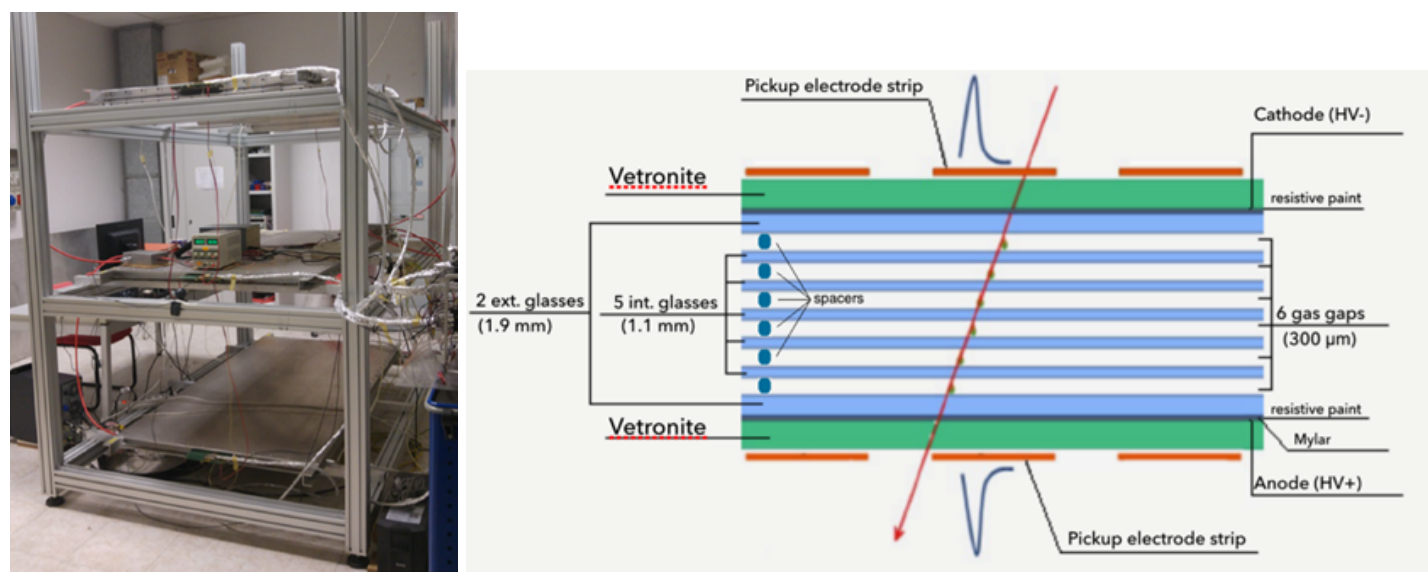

Figure 2: Left. Picture of one of the EEE telescopes, consisting in three MRPC chambers. Right. Sketch of the inner structure of a MRPC.

Data taking is organized in Runs which follow the school year, from October to June, as schematically 
shown in Fig. 3. In the last years, over a hundred billion events have been collected. Since 2017, the EEE network has undergone an upgrade process, which includes the construction of over 50 new chambers, with $250 \mu \mathrm{m}$ gaps that allow a lower operating voltage, and the test for new eco-friendly gas mixtures for the MRPCs.

After the assembly of the chambers, the telescopes are sent and installed in Italian highschools, where students and teachers, with the supervision of EEE researchers, take care of the commissioning of the station and of the start of data taking operations. Finally, students are responsible for the daily monitoring, reporting data acquisition conditions into an online e-log [16] (weather parameters, HV and current settings, acquisition rate, etc.), and take care of the maintenance of the telescopes.

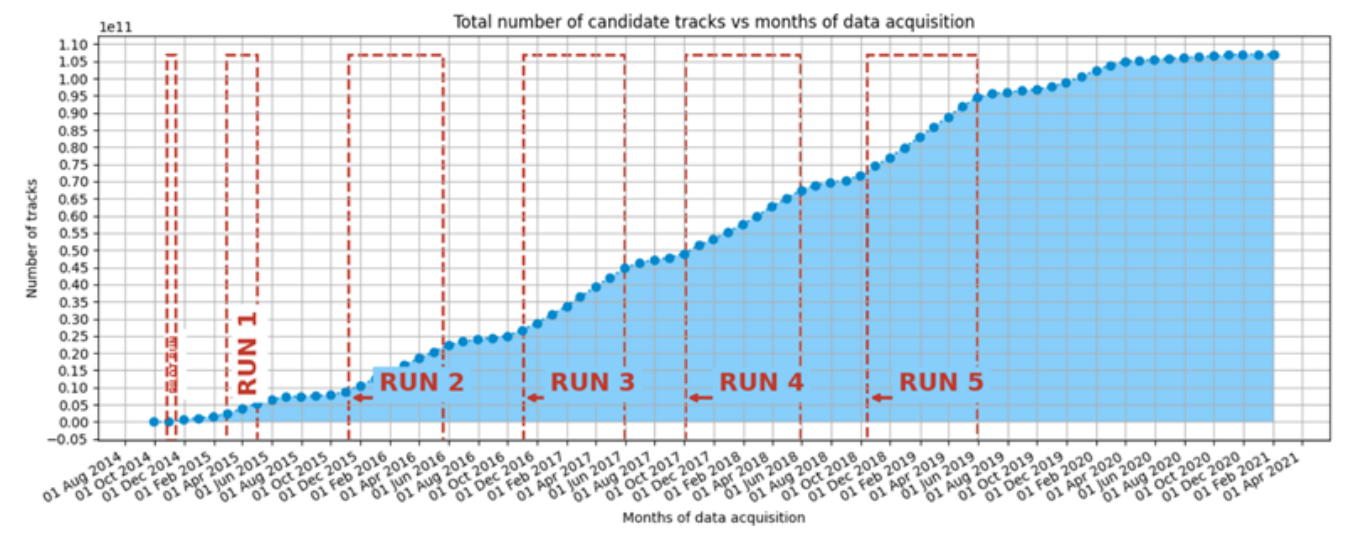

Figure 3: Organized data taking of EEE telescopes arranged in Runs, since 2014.

\section{Outreach activities}

The students of the project are involved in local and international outreach activities, such as the International Cosmic Day (ICD) [17] or the European Researcher's Night (ERN) [18], with the aim of heighten young people's interest in a career in science. In November students take part to the ICD, which is an outreach event for astroparticle physics organized by DESY in cooperation with Netzwerk Teilchenwelt, IPPOG, QuarkNet, Fermilab, INFN and Centro Fermi. During this day, groups of high-school students and teachers meet scientists to learn about cosmic rays and perform together an experiment with cosmic particles. The students are pushed to work together like in an international collaboration, discussing their results in joint video conferences where scientists give lectures providing an insight into the current astroparticle physics research. In Fig. 4 a collage of pictures taken during last in-person ICD are shown: students participated either from their highschools or from Universities and research centres involved in the project. A similar spirit guides also the ERN, a research communication and promotion event taking place in Europe with the aim of promoting excellence research projects across Europe and beyond, increasing awareness among the general public of the importance and benefits of research and innovation and spreading science among a young public.

During the bi-annual conferences held at Ettore Majorana Foundation And Centre For Scientific Culture in Erice, students have the possibility to take part to real time experiments with atmospheric 

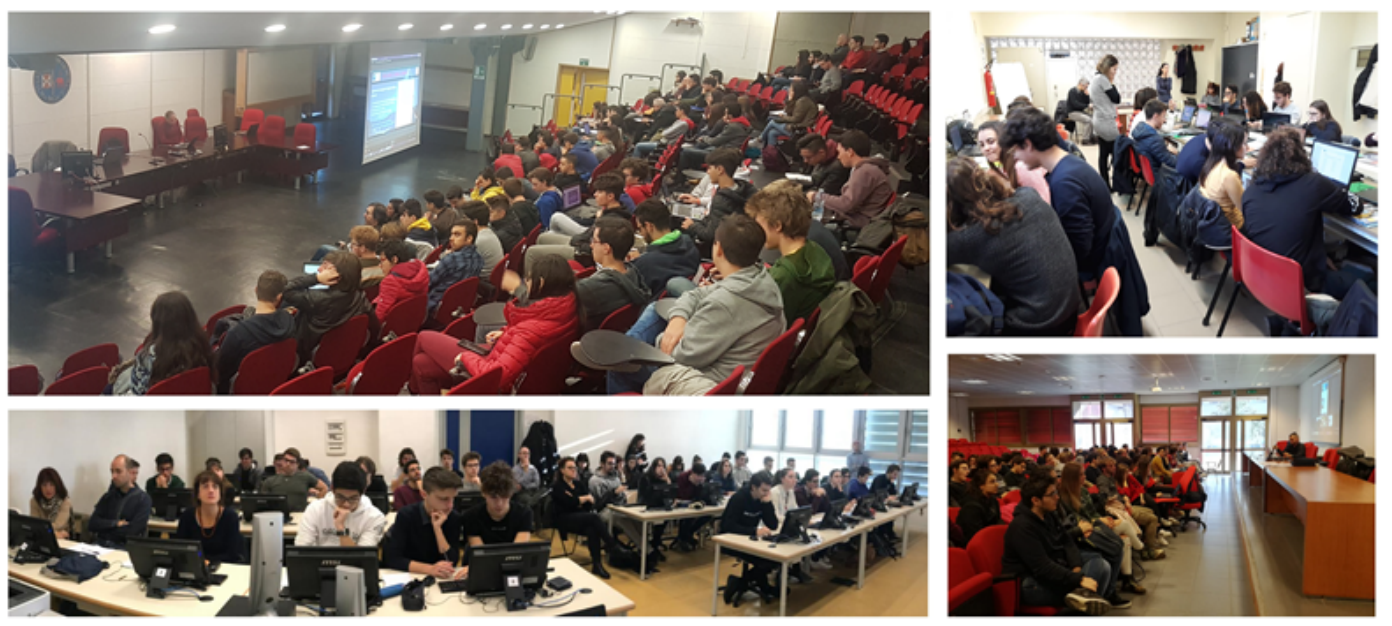

Figure 4: Collage of pictures taken during last in-person International Cosmic Day, capturing students while working on the data analysis of a cosmic ray experiment.

muons. It is worth mentioning two interesting measurements that have been carried out in such occasions: the measurement of the cosmic flux variations with altitude [19] and the measurement of the Earth radius [20]. In the first case, a measurement of the cosmic ray flux was carried out from the sea level up to an altitude of $760 \mathrm{~m}$, from Castellammare del Golfo to Erice, in Sicily. The experimental setup consisted of a set of 15 portable small-size scintillator-based detectors, the so-called Cosmic Boxes, as shown in Fig. 5. The students were divided in groups to measure the cosmic ray flux in the different locations (see Fig. 6), collecting the data over 30-minutes intervals. The count rate, together with the environmental temperature and pressure, were annotated by the students and finally presented during the conclusive lecture of the conference. The resulting variation in percentage of the cosmic ray flux with altitude was $26 \pm 2 \% / \mathrm{km}$, in fair agreement with the existing literature.
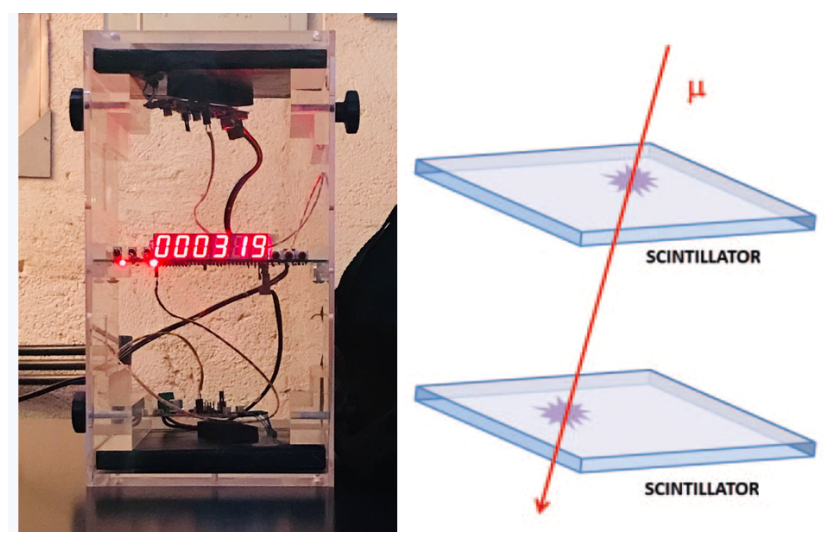

Figure 5: Sketch (on the right) and picture (on the left) of the Cosmic Box used to carry out the measurement of the cosmic ray variation with altitude.

Concerning the measurement of the Earth radius, an experiment using the Eratostene method was carried out. Such experiment took place between Erice and several cities in the northern Italy. 
The experimental setup was: only a wooden pole, a bubble level, a set square and a measuring tape. Measuring the length of the shadow $\left(\mathrm{L}_{S}\right)$ of a wooden pole (with a length $\mathrm{L}_{p}$ ) on a flat floor, when the Sun is at zenith, in two different places (A and B) on the same meridian separated by a distance $d_{A B}$, it is possible to estimate the Earth radius $\left(\mathrm{R}_{\text {Earth }}\right)$ by comparing the incident angle $\omega$ of the Sun light with respect to zenith in $\mathrm{A}$ and $\mathrm{B}$, since $d_{A B}=\omega \times R_{\text {Earth }}$ and $\omega=\arctan \left(\mathrm{L}_{\mathrm{S}} / \mathrm{L}_{\mathrm{p}}\right)$. The students attending the conference in Erice were divided into small groups to performe the measurement when the Sun was at the zenith, as shown in Fig. 7. Simultaneously, other students performed similar measurement in their cities (Milano, Lodi, Torino and Treviso). The outcome of the measurements of each group of students was presented during the conclusive lecture of the conference. As a result, four estimates of the Earth radius were obtained and found in fair agreement with the Eratostene result. The results of such experiments have been published including the participating high-school students and teachers in the author list.
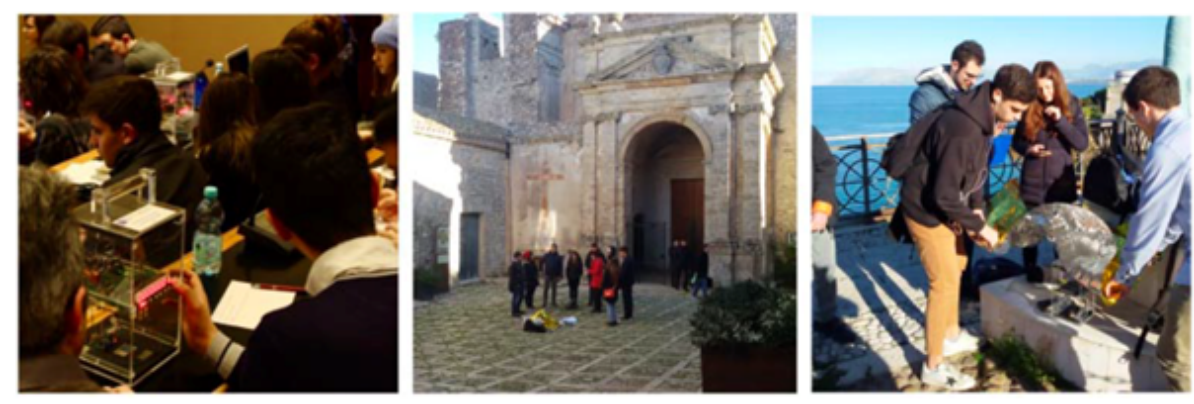

Figure 6: Pictures of students carrying out the measurements with a Cosmic Box during the masterclass (on the left), in Erice (in the middle) and in Castellammare del Golfo (on the right).

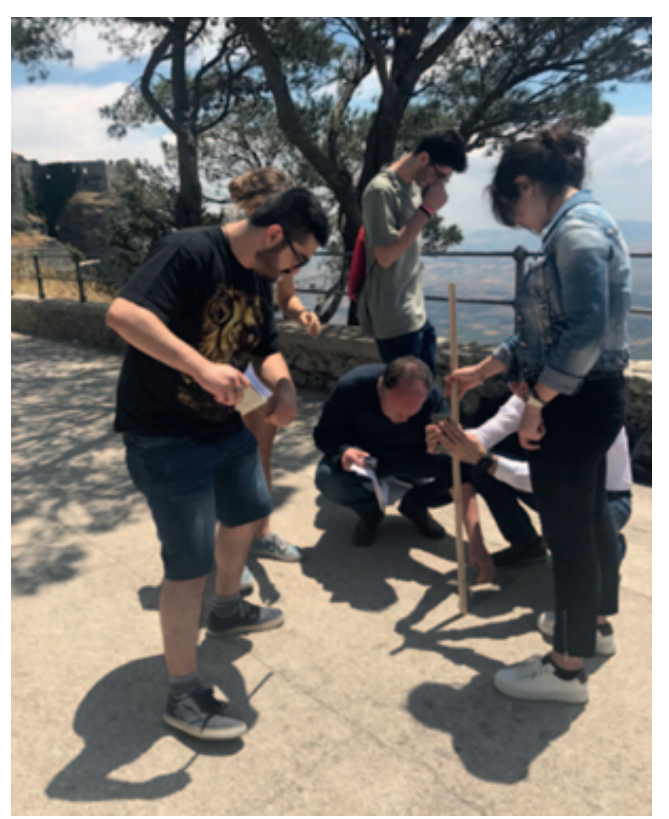

Figure 7: Picture of students carrying out the measurement of the Earth radius using the Eratostene method in Erice. 


\section{The PolarQuEEEst project}

Another project strictly related to EEE, is the PolarquEEEst experiment, set to merge a scientific project with the outreach mission. Its scientific program consists in a series of measurements of the cosmic ray flux up to the far North latitudes. The project started in Summer 2018, when three telescopes made out of scintillators were built by high-school student teams at CERN and installed in Italy, Norway and on a sailing boat leaving from North Iceland, to circumnavigate the Svalbard archipelago. The trip was carried out within the Polarquest project 1928-2018 [21], an expedition to commemorate the $90^{\text {th }}$ anniversary of the unfortunate airship ITALIA mission. The PolarquEEEst project continued afterwards with a series of measurements in Italy, southward reaching Lampedusa, and in Germany. In 2019, the PolarquEEEst experiment installed a cosmic ray observatory for the detection of secondary cosmic muons at $\mathrm{Ny}$ Alesund, at $79^{\circ} \mathrm{N}$, made of three independent identical detectors positioned a few hundred meters from each other. The detectors were synchronized in order to operate together as a network, complementing the cosmic neutron detection stations at the Svalbard archipelago.

\section{Summary}

The Extreme Energy Events is an experiment to study high-energy cosmic rays with an innovative outreach approach. High-school students are directly involved in the experiment, playing a primary role starting from the detector construction at CERN to the data taking and analysis. About 200 MRPCs have been built by students and installed in Italian high-schools, over the past years. The EEE project is still expanding with the aim of enlarging the network and involving more schools. The EEE experiment includes many educational and outreach activities, through meetings and conferences, allowing the students to be involved in the professional scientific research. Moreover, students are involved in local and international outreach activities, such as the European Researcher's Night or the International Cosmic Day, with the aim of heighten young people's interest in science and research careers.

\section{References}

[1] EEE web site: https://eee.centrofermi.it/

[2] EEE Collaboration, Looking at the sub-TeV sky with cosmic muons detected in the EEE MRPC telescopes, Eur. Phys. J. Plus 130 (2015) 187.

[3] EEE Collaboration, First detection of extensive air showers with the EEE experiment, Nuovo Cimento 125 B (2010) 243.

[4] EEE Collaboration, Time Correlation measurements from extensive air showers detected by the EEE telescopes, Eur. Phys. J. Plus (2013) 128, 148.

[5] EEE Collaboration, A study of upward-going particles with the EEE telescopes, NIM A 816 (2016) 142. 
[6] EEE Collaboration, Time and orientation long distance correlations between extensive air showers detected by the MRPC telescopes of the EEE Project, Nuovo Cimento C40(2017)196.

[7] EEE Collaboration, Search for long distance correlations between extensive air showers detected by the EEE network, Eur. Phys. J. Plus (2018) 133: 34.

[8] EEE Collaboration, Performance of a six gap MRPC built for large area coverage, NIM A 593 (2008) 263.

[9] EEE Collaboration, Recent results and performance of the multi-gap resistive plate chambers network for the EEE Project, JINST 11 (2016) C11005.

[10] EEE Collaboration, The cosmic muon and detector simulation framework of the extreme energy events (EEE) experiment, Eur. Phys. J. C (2021) 81:464.

[11] EEE Collaboration, Test of new eco-gas mixtures for the multigap resistive plate chambers of the EEE project, NIM A 936 (2019) 493.

[12] EEE Collaboration, Observation of the February 2011 Forbush decrease by the EEE telescopes, Eur. Phys. J. Plus (2011) 126, 61.

[13] EEE Collaboration, Results from the observation of Forbush decreases by the Extreme Energy Events experiment, Proceedings of the ICRC2015 Conference, PoS(ICRC2015)097.

[14] EEE Collaboration, The EEE MRPC telescopes as traking tools to monitor building stability, JINST 14 (2019) C05022.

[15] EEE Collaboration, The Extreme Energy Events experiment: an overview of the telescopes performance, JINST 13 (2018) P08026.

[16] E-log web site: https://iatw.cnaf.infn.it/eee/elog/

[17] ICD web site: https://icd.desy.de/

[18] ERN web site: https://ec.europa.eu/research/mariecurieactions/actions/msca-citizens

[19] EEE Collaboration, FISICA PER TUTTI - Come varia il flusso dei raggi cosmici con la quota? Basta chiederlo agli studenti del progetto EEE - How does cosmic ray flux vary with altitude? Let's ask it to EEE project students, Giornale di Fisica, VOL. LIX, N. 3, Luglio - Settembre 2018.

[20] EEE Collaboration, Gli studenti del progetto EEE sulle orme di Eratostene per la misura del raggio della Terra, Giornale di Fisica 60 (2019) 107.

[21] Polarquest 2018 web site: http://www.polarquest2018.org/ 


\section{Full Authors List: EEE Collaboration}

M. Abbrescia ${ }^{1,2}$, C. Avanzini ${ }^{3}$, L. Baldini ${ }^{3,4}$, R. Baldini Ferroli ${ }^{5}$, G. Batignani ${ }^{3,4}$, M. Battaglieri ${ }^{6,7}$, S. Boi ${ }^{8,9}$, E. Bossini ${ }^{3,4}$, F. Carnesecchi ${ }^{10,11}$, C. Cicalò ${ }^{9}$, L. Cifarelli ${ }^{10,11}$, F. Coccetti ${ }^{12}$, E. Coccia ${ }^{13}$, A. Corvaglia ${ }^{14}$, D. De Gruttola ${ }^{15,16}$, S. De Pasquale ${ }^{15,16}$, F. Fabbri ${ }^{5}$, L. Galante ${ }^{17,18}$, M. Garbini ${ }^{10,12}$, G. Gemme ${ }^{6}$, I. Gnesi ${ }^{12,19}$, S. Grazzi ${ }^{6,20}$, D. Hatzifotiadou ${ }^{10,21}$, P. La Rocca ${ }^{22,23}$, Z. $\mathrm{Liu}^{24}$, G. Mandaglio ${ }^{20,23}$, G. Maron ${ }^{25}$, M. N. Mazziotta ${ }^{2}$, A. Mulliri ${ }^{8,9}$, R. Nania ${ }^{10}$, F. Noferini ${ }^{10}$, F. Nozzoli ${ }^{26}$, F. Palmonari ${ }^{10,11}$, M. Panareo ${ }^{14,27}$, M. P. Panetta ${ }^{12,14}$, R. Paoletti ${ }^{3,28}$, C. Pellegrino ${ }^{25}$, O. Pinazza ${ }^{10}$, C. Pinto ${ }^{22,23}$, S. Pisano ${ }^{5,12}$, F. Riggi ${ }^{22,23}$, G. C. Righini ${ }^{29}$, C. Ripoli ${ }^{15,16}$, M. Rizzi ${ }^{2}$, G. Sartorelli ${ }^{10,11}$, E. Scapparone ${ }^{10}$, M. Schioppa ${ }^{19,30}$, A. Scribano ${ }^{28}$, M. Selvi ${ }^{10}$, G. Serri ${ }^{8,9}$,

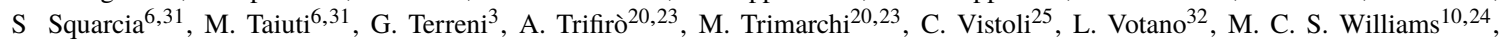
A. Zichichi ${ }^{10,11,21}$ and R. Zuyeuski ${ }^{24}$

${ }^{1}$ Dipartimento Interateneo di Fisica, Università di Bari, Bari, Italy. ${ }^{2}$ INFN Sezione di Bari, Bari, Italy. ${ }^{3}$ INFN Sezione di Pisa, Pisa, Italy. ${ }^{4}$ Dipartimento di Fisica, Università di Pisa, Pisa, Italy. ${ }^{5}$ INFN Laboratori Nazionali di Frascati, Frascati (Rome), Italy. ${ }^{6}$ INFN Sezione di Genova, Genova, Italy. ${ }^{7}$ Thomas Jefferson National Accelerator Facility, Newport News, VA, USA. ${ }^{8}$ Dipartimento di Fisica, Università di Cagliari, Cagliari, Italy. ${ }^{9}$ INFN Sezione di Cagliari, Cagliari, Italy. ${ }^{10}$ INFN Sezione di Bologna, Bologna, Italy. ${ }^{11}$ Dipartimento di Fisica ed Astronomia, Università di Bologna, Bologna, Italy. ${ }^{12}$ Museo Storico della Fisica e Centro Studi e Ricerche "E. Fermi", Rome, Italy. ${ }^{13}$ Gran Sasso Science Institute, LAquila, Italy. ${ }^{14}$ INFN Sezione di Lecce, Lecce, Italy. ${ }^{15}$ Dipartimento di Fisica, Università di Salerno, Salerno, Italy. ${ }^{16}$ INFN Gruppo Collegato di Salerno, Salerno, Italy. ${ }^{17}$ Dipartimento di Scienze Applicate e Tecnologia, Politecnico di Torino, Torino, Italy. ${ }^{18}$ INFN Sezione di Torino, Torino, Italy. ${ }^{19}$ INFN Gruppo Collegato di Cosenza, Cosenza, Italy. ${ }^{20}$ Dipartimento di Scienze Matematiche e Informatiche, Scienze Fisiche e Scienze della Terra, Università di Messina, Messina, Italy. ${ }^{21} \mathrm{CERN}$, Geneva, Switzerland. ${ }^{22}$ Dipartimento di Fisica e Astronomia, Università di Catania, Catania, Italy. ${ }^{23}$ INFN Sezione di Catania, Catania, Italy. ${ }^{24}$ ICSC World laboratory, Geneva, Switzerland. ${ }^{25}$ INFN-CNAF, Bologna, Italy. ${ }^{26}$ INFN Trento Institute for Fundamental Physics and Applications, Trento, Italy. ${ }^{27}$ Dipartimento di Matematica e Fisica, Università del Salento, Lecce, Italy. ${ }^{28}$ Dipartimento di Scienze Fisiche, della Terra e dell'Ambiente, Università di Siena, Siena, Italy. ${ }^{29}$ CNR Istituto di Fisica Applicata "Nello Carrara", Sesto Fiorentino (Florence), Italy. ${ }^{30}$ Dipartimento di Fisica, Università della Calabria, Rende (Cosenza), Italy. ${ }^{31}$ Dipartimento di Fisica, Università di Genova, Genova, Italy. ${ }^{32}$ INFN, Laboratori Nazionali del Gran Sasso, Assergi (L'Aquila), Italy. 